\title{
Fertilizer Effects on Nutrient Elements, Total Polyphenols and Anti-oxidant Contents of Roselle (Hibiscus sabdariffa) Leaves
}

\author{
Chin Sue ${ }^{1,2}$, Yadong Qi $^{1^{*}}$, Kit L Chin ${ }^{1}$, Vanessa A Ferchaud ${ }^{1}$, Michael Breithaupt ${ }^{2}$ and Roosevelt Payne ${ }^{1}$ \\ ${ }^{1}$ Southern University Agricultural Research \& Extension Center, Land Grant Campus, Southern University and A\&M College, USA
}

${ }^{2}$ School of Plant and Environmental and Soil Sciences, Louisiana State University, USA

Received: 制 April 24, 2018; Published: 㘹 May 08, 2018

*Corresponding author: Yadong Qi, Urban Forestry Program, Southern University land Grant Campus, Southern University and A\&M College, Baton Rouge, LA 70813

\begin{abstract}
A great deal of research has been geared toward the calyces of roselle due to their medicinal properties. Though leaves of roselle have been consumed by diversed ethnic groups, little research efforts have been focused on leaves due to unestablished beneficial bioactivities. This paper provides preliminary data on the effect of various types and rates of fertilizer on fresh fruit yields, total polyphenol and antioxidant contents of the leaves of roselle (Senegal accession). Fertilizer applications at planting time had nonsignificant increasing effect on fresh fruit yield/plant and non-significant reduction in total polyphenol and antioxidant contents of the leaves. Inconsistent effects of applied fertilizer on macro and micro nutrient contents of the leaves were observed. In general, $\mathrm{N}, \mathrm{P}, \mathrm{K}, \mathrm{Ca}, \mathrm{Mg}, \mathrm{S}, \mathrm{B}, \mathrm{Mn}$ and $\mathrm{Zn}$ were significantly $(\mathrm{P}<0.05)$ increased by selective fertilizer types and rates while $\mathrm{Cu}$ and Fe contents were not significantly affected by fertilization. Further studies on fertilization along with increased frequencies of application are needed to validate these findings.
\end{abstract}

Keywords: Roselle; Hibiscus sabdariffa; Fertilizer; Phenols; Antioxidants and fruit yield

\section{Introduction}

More than 300 species of Hibiscus species (Malvacea family) can be found growing in both tropical and subtropical regions of the world [1]. Roselle, Hibiscus sabdariffa L., is only one of the hibiscus species that has its sepals of the flower developed into a swollen red calyx enclosing the mature fruit at maturity. The calyces are found to be rich in nutrients and photochemical compounds that exhibits anti-oxidant activities Consequently, economic interests have been mainly focused on roselle calyces which are used worldwide in the production of herbal tea, cold refreshing drinks, jellies, jam, sauces, chutneys, wines, preserves and food colorants (D'Heureux-Calix and Badrie, 2004). Because of the folk's medicinal uses, a great deal of research has been geared toward roselle calyces. Most research has been heavily directed toward the studies of phytochemistry, anti-oxidant properties and effects of calyx extracts on anti-oxidant activities, and other bioactivities such as anti-inflammatory action, anti-microbial, and antitumor activities [2] and Wang 2015. Even though leaves are widely consumed by a diverse population of ethnic groups, particularly in tropical regions of Africa, little research effort has been focused on leaves. In recent studies [2], roselle leaves are found to be a good source of phenolic acids and flavonoids that exhibit anti-oxidant activities. The purpose of this preliminary study is to evaluate the effect of various types and rates of fertilizer application on roselle fruit yield, nutritional, phenolic and antioxidant content of the leaves.

\section{Materials and Methods}

\section{Chemicals and reagents}

ABTS (2,2'-azino-bis (3-ethylbenzothiazoline-6-sufonic acid), potassium persulfate were purchased from Sigma-Aldrich of St. Louis, MO, USA. Folin Ciocalteu's phenol reagent was purchased 
from MP Biomedicals of Solon, OH, USA. Water and methanol were purchased from Fisher Scientific of Fair Lawn, BJ, USA.

\section{Experiment and plant samples}

A field experiment was conducted to evaluate the effects of the applied fertilizers at planting time on roselle plants of Senegal accession during the 2014 growing season at Southern University's research farm, Baton Rouge, Louisiana which has a subtropical climate $(30.5240 \mathrm{~N}$ and $91.1900 \mathrm{~W}$ and elevation of $22 \mathrm{~m})$. The area has an average precipitation of $1700 \mathrm{~mm}$ and the average annual temperature is $19.70 \mathrm{C}$. The field has a silty clay loam soil with a $\mathrm{pH}$ of 7.1. The seeds of Senegal accession were planted in the $2 \times 2$ cell-pack trays in the greenhouse at the beginning of April, 2014. Seedlings were hand-transplanted into the field one month after germination. A completely randomized design with a total nine applications as treatments. These treatments were the control; 3, 6 and $9 \#(\mathrm{lb}=454 \mathrm{~g}) / 30.48 \mathrm{~m}$. of organic fertilizer 4-2-2, respectively; 3,6 and 9\# (454g)/30.48m of organic fertilizer 8-5-5, respectively; and 3 and $6 \#(\mathrm{lb}=454 \mathrm{~g}) / 30.48 \mathrm{~m}$. of inorganic fertilizer 13-13-13, respectively. Each treatment consisted of 16 plants spaced at 6 feet $(1.83 \mathrm{~m})$ between plants within a $3.5 \mathrm{ft}(1.07 \mathrm{~m})$ row. Between row spacing was 8 feet $(2.44 \mathrm{~m}$.). Fertilizers were applied and incorporated on top of the raised bed. Seedlings were planted one day after fertilizer applications. Leaves were randomly collected in late July from branches at waist height from 10 plants of each treatment.

\section{Nutrient elemental analysis}

Leaves in the brown paper bags were dried at 500C in the Precision Thelco Laboratory oven for 8 hours in the oven and then ground using Thomas-Wiley Model 4 grinder with a 30 mesh screen. The ground samples in the vials were subject to one-hour of drying before they were stored in the desiccator to prevent moisture contamination. Three individual $0.5 \mathrm{~g}$ of composite ground leaf samples from each treatment were placed in 50mL Digi-tubes (SCP Sciences) and were digested using DEENA automatic digester. Each digested sample in the Digi tube was brought up to a total volume of $20 \mathrm{~mL}$ with distilled water. The solution with digested sample was vacuum-filtered with a 1.0 micron Digi-filter. The filtrate was analyzed by SPECTRO ARCOS ICAP for $\mathrm{Al}, \mathrm{Bo}, \mathrm{Ca}, \mathrm{Cu}, \mathrm{Fe}, \mathrm{Mg}$, Mn, Mo, P, K, NA, S and Zn. Separately, $0.15 \mathrm{~g}$ of dried leaf sample was used for determining the amount of carbon and nitrogen using LECO CN Analyzer.

\section{Sample preparation for total phenol and antioxidant activity determination}

The method of sample preparation was adopted from Xu [3] and Zhen [2]. A 100mg of each dried and ground leaf sample was measured and placed in a volumetric flask. Twenty-five $\mathrm{mL}$ of $70 \%$ (v/v) methanol/water with $0.1 \%$ acetic solution was added into the flask. The sample extraction was then put in ultrasonic water bath for 10 minutes followed by shaking at room temperature for overnight. The extract of each sample was then filtered through 0.45um filter. The filtrate was used for the Folin-Ciocalteu and ABTS radical scavenging assays.

\section{Phenolic content determination (Folin-Ciocalteu assay)}

The Folin-Ciocalteu assay with modifications [4] used by Zhen [2] was adopted for the determination of the total phenolic content of the leaves. $40 \mathrm{ul}$ of the prepared leaf extract was mixed with 900ul diluted Folin Ciocalteu's reagent followed by incubation at room temperature for 5 minutes. Then, $400 \mathrm{ml}$ of $15 \%$ sodium carbonate was added. The mixture was allowed to react at room temperature for 45 minutes. The UV absorption of the mixture solution at wavelength of $752 \mathrm{~nm}$ was measured against a blank solution. The standard curve was measured based on the prepared gallic acid standard solution $(0.38,0.19,0.095$ and $0.475 \mathrm{mg} / \mathrm{ml})$. The result was transformed as $\mathrm{mg} / \mathrm{g}$ of gallic acid. The results were calculated from the mean of three replicates.

\section{In vitro antioxidant activity (ABTS radical scavenging assay)}

The procedure of the decolonization of the ABTS radical cation [5] used by Zhen [2] was adopted for the determination of antioxidant activity. $38.4 \mathrm{mg}$ of ABTS and $6.6 \mathrm{mg}$ of potassium per sulfate were co-dissolved in $10 \mathrm{ml}$ of water and stored for 16 hours in the dark environment to form stable radical action (ABTS+). The stored radical solution was then diluted using ethanol to a concentration with UV absorption of $0.70+/-0.20$ at $734 \mathrm{~nm}$. $900 \mathrm{ul}$ of the diluted ABTS radical working solution was mixed with $10 \mathrm{ul}$ of the leaf extract followed by 20 minutes of reaction at room temperature. The decolonization of the mixed solution indicates that antioxidant compounds in the extract quenched ABTS radical actions. There was a quantitative relationship between the reduction absorbance at $734 \mathrm{~nm}$ and the concentration antioxidants present in the sample [2]. The standard curve was built up by plotting the concentrations of Trolox against the percentage of inhibition. The value of antioxidant capacity of the sample was calculated as Trolox equivalent antioxidant capacity. The final result was expressed as the $\%$ of antioxidant per dry weight basing on the calculation from the mean of three replicates.

\section{Results and Discussion}

Figure 1 shows the effects of the type of fertilizer and its rate on fresh fruit yield/plant. There was a trend to indicate that applied fertilizers increased fresh fruit yield of roselle of Senegal accession under this study. However, the yield increases were observed to be non-significant. Since all fertilizers were applied at the planting time in spring, the effect of fertilization might have faded away during the late summer and early fall during which fruits were produced. This fading effect of applied fertilizers can be supported by various extension publications indicating the recommended side-dressing of fertilizers are needed for various vegetable crops during the growth period [6-8]. Figure 2 shows the effects of the type and rate of fertilizer applied at planting time on the total polyphenol and antioxidant content of roselle leaves in \% of dry 
weight. There appeared to have a trend that increased amounts and rates of fertilizer exhibited a reduction in total polyphenol content. The reduction in polyphenol content might be due to less nutritional stress by plants receiving increased amount of fertilizer.
It is hypothesized here that further application of fertilizers to enhance plant growth and yield may cause significant reduction in polyphenol production. It had been reported that plants under stress provided more antioxidants in response to stresses [9-12].

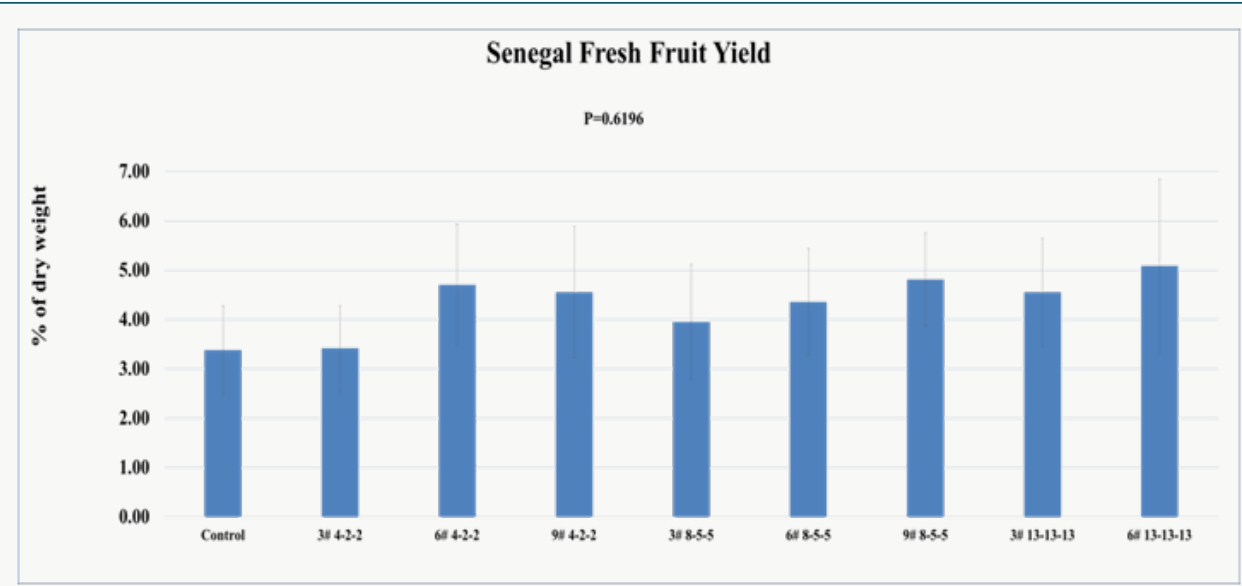

Figure 1: Effects of the type and rate of fertilizer applied at planting time on fresh roselle fruit yield in $\mathrm{kg} / \mathrm{plant}$. Note: \# is denoted as lb. (454 g) of fertilizer applied per 100 feet $(30.48 \mathrm{~m})$ row.

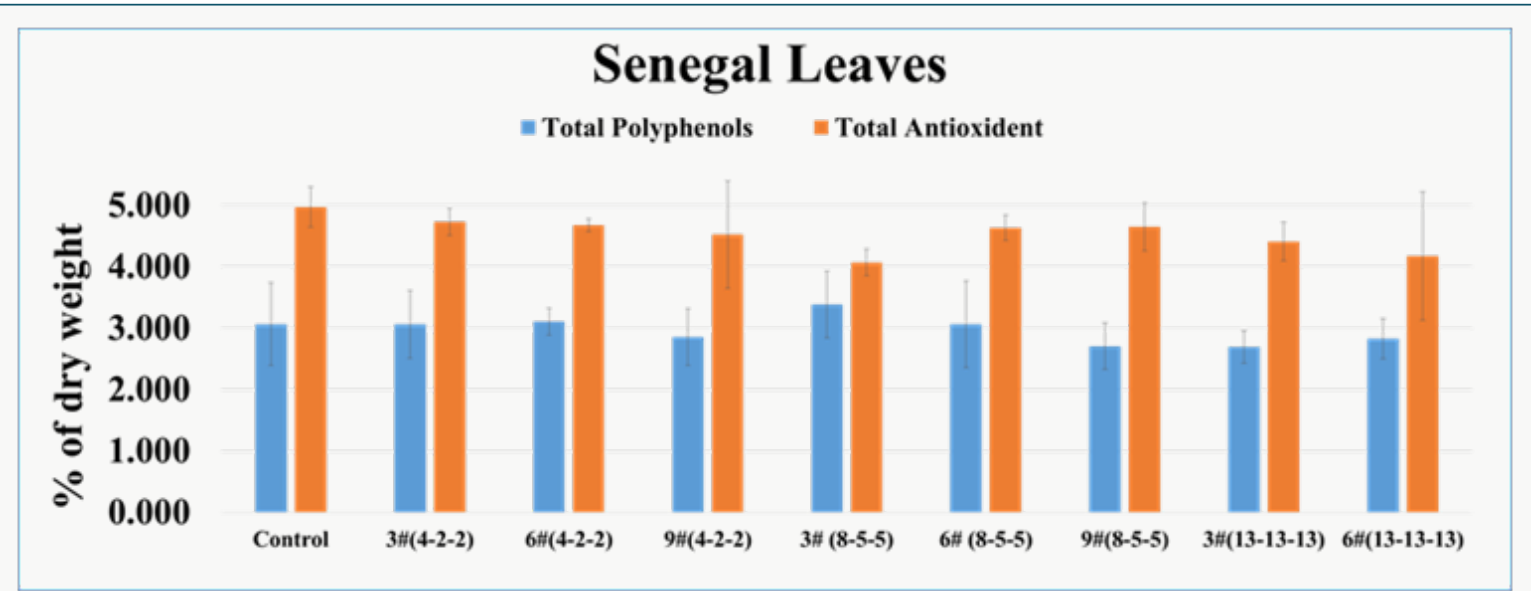

Figure 2: Effects of the type and rate of fertilizer applied at planting time on total polyphenol and antioxidant contents of roselle leaves in \% of sample dry weight. Note: \# is denoted as lb. (454 g) of fertilizer applied per 100 feet (30.48 m) row.

Figure 3 shows the effects of the type and rate of fertilizer applied at planting time on (A) macro-nutrients and (B) micro-nutrients of roselle leaves. The results when the effects were compared to that of the Control treatment, showed that (a) 3\# of organic fertilizer 4-2-2 and 3\#, 6\# and 9\# of organic fertilizer significantly $(\mathrm{P}<0.05)$ increased $\mathrm{N}$ content of the leaves, (b) all fertilizer treatments had no significant effect on P content of the leaves, (c) 9\# of organic fertilizer 4-2-2 significantly $(\mathrm{P}<0.05)$ increased $\mathrm{K}$ content of the leaves, (d) all fertilizer treatments had no significant effect on $\mathrm{Ca}$ content of the leaves, (e) 9\# of organic fertilizer 4-2-2, 6\# and 9\# of organic fertilizer 8-5-5 significantly $(\mathrm{P}<0.05)$ increased $\mathrm{Mg}$ content of the leaves, (f) $3 \#, 6 \#$ and $9 \#$ of organic fertilizer and $6 \#$ of inorganic fertilizer $13-13-13$ significantly $(\mathrm{P}<0.05)$ increased $\mathrm{S}$ content of the leaves, ( $\mathrm{g}$ ) all fertilizer treatments had no significant effect on $\mathrm{Cu}$ and $\mathrm{Fe}$ contents of the leaves, (h) 3\# and 6\# of organic fertilizer significantly $(\mathrm{P}<0.05)$ increased $\mathrm{B}$ content of the leaves, (i) $3 \#$ and $9 \#$ of organic fertilizer $4-2-2$, 9\# of organic fertilizer
8-5-5 and 3\# and 6\# of inorganic fertilizer 13-13-13 significantly increased Mn content of the leaves and (j) 9\# of organic fertilizer 4-2-2, 3\#, 6\# and 9\# of organic fertilizer 8-5-5 and 6\# of inorganic fertilizer significantly increased $\mathrm{Zn}$ content of the leaves. The results of this study showed inconsistent in nutrient elemental contents of plants in response to fertilizer treatments. In general, $\mathrm{N}, \mathrm{P}, \mathrm{K}, \mathrm{Ca}, \mathrm{Mg}$, $\mathrm{S}, \mathrm{B}, \mathrm{Mn}$, and $\mathrm{Zn}$ were significantly increased by selective fertilizer types and rates while $\mathrm{Cu}$ and Fe were not significantly affected by the fertilizer applications.

\section{Conclusion}

Fertilizer applications at planting time enhanced many macro and micro nutrient elemental contents of roselle leaves but did not adequately affect fresh fruit yield, leaf total phenol and anti-oxidant contents. Reviewed literature provides evidence a significant increase in fruit yield can be obtained through an increase in fertilizer application as side-dressing. Evidence provided by 
literatures also suggests that an increase in fertilizer applications which reduce nutritional stress can also possibly reduce polyphenol content and antioxidant activity of roselle. Inconsistent results of this study suggest that further studies on the effect of fertilizer and the frequency of fertilizer application are needed to validate the results of these findings and conclusion.

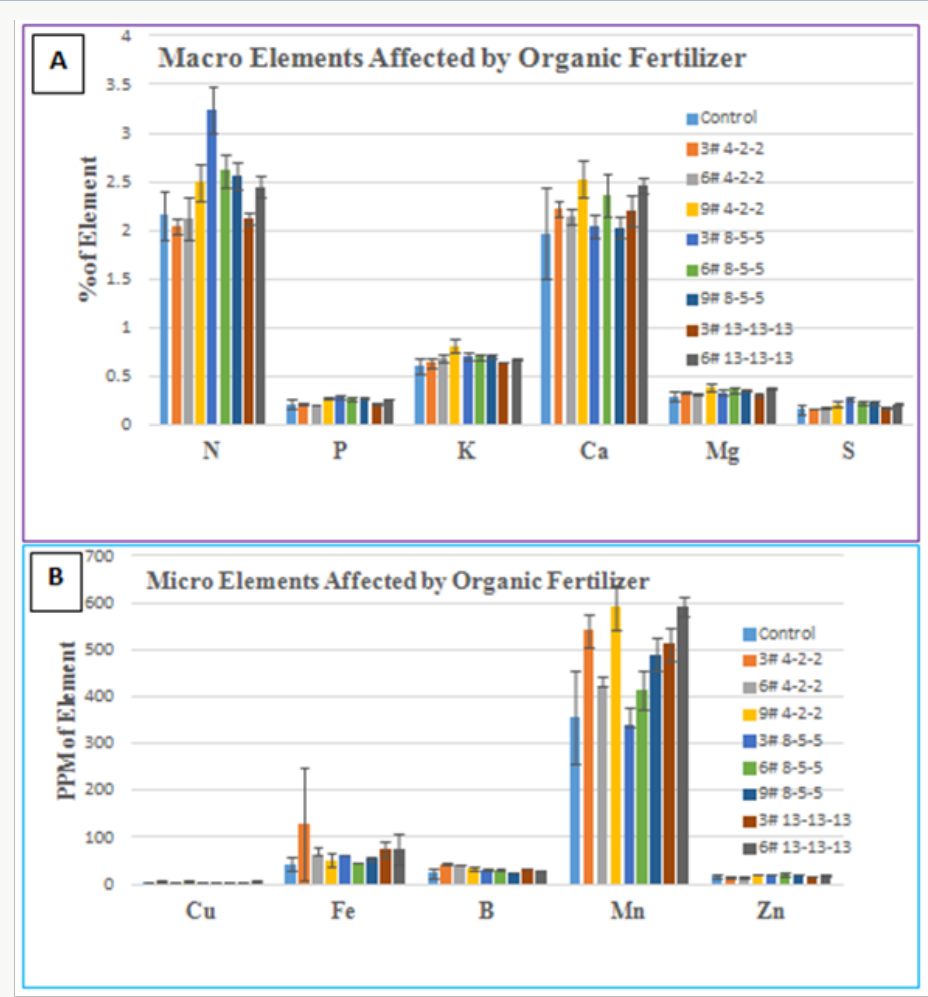

Figure 3: Effects of the type and rate of fertilizer applied at planting time on (A) macro-nutrients and (B) micro-nutrients of roselle leaves. Note: \# is denoted as $\mathrm{lb}$. (454 g) of fertilizer applied per 100 feet $(30.48 \mathrm{~m})$ row. Macro-nutrients are expressed in $\%$ of dry weight while micro-nutrients are expressed in PPM in dry weight.

\section{References}

1. ASNAPP (2003) Market survey: Hibiscus sabdariffa by Agribusiness in Sustainable Natural African Plant Products (ASNAPP).

2. Zhen J, TS Villani, Y Guo, Y Qi, K Chin, et al. (2016) Phytochemistry, antioxidant, total phenolic content and anti-inflammatory activitity of Hibiscus sabdariffa leaves. Food Chemistry 190: 673-680.

3. Xu YP, JE Simon, C Welch, JD Wightman, MG Ferruzzi, et al. (2011) Survey of polyphenol constituents in grapes and grape derived products. Journal of Agricultural and Food Chemistry 59(19): 10586-10593.

4. Singleton VL (1985) Citation classic-Colorimetry of total phenolins with phosphomolybdic-phosphotungstic acid reagents. Current Contents/ Agriculture, Biology Environmental Science 48: 18.

5. Re R, N Pellegrini, A Proteggente, A Pannala, M Yang, et al. (1999) Antioxidant activity applying an improved ABTS radical cation decolorization assay. Free Radical Biology \& medicine 26(9-10): 12311237.
6. Lane Mick (1998) Sidedressed Manure Benefits Soybeans. Corn and Soybean Digest.

7. Greg Arnold (2014) Side-dressing Manure into Corn has Promising Results. Ohio's Country Journal.

8. White Joe (2018) Side dressing some vegetable crops can boost yields

9. Mierziak J, K Kostyn, A Kulma (2014) Flavonoids as important molecules of plant interactions with the environment. Molecules 19(10): 1624016265.

10. Michalak A (2006) Phenolic compounds and their antioxidant activity in plants growing under heavy metal stress. Olish J of environmental studies 15(4): 523-530.

11. Rivero RM, JM Ruiz, PC Garcia, LR Lopez Lefebre, E Sanchez, et al. (2001) Resistance to cold and heat stress: accumulation of phenolic compounds in tomato and watermelon plants. Plant Science 160(2): 315-321.

12. Winkel Shirley B (2002) Biosynthesis of flavonoids and effects of stress. Current Opinion in Plant Biology 5(3): 218-223. 
(c) This work is licensed under Creative To Submit Your Article Click Here:

Submit Article

DOI: 10.32474/CIACR.2018.02.000142

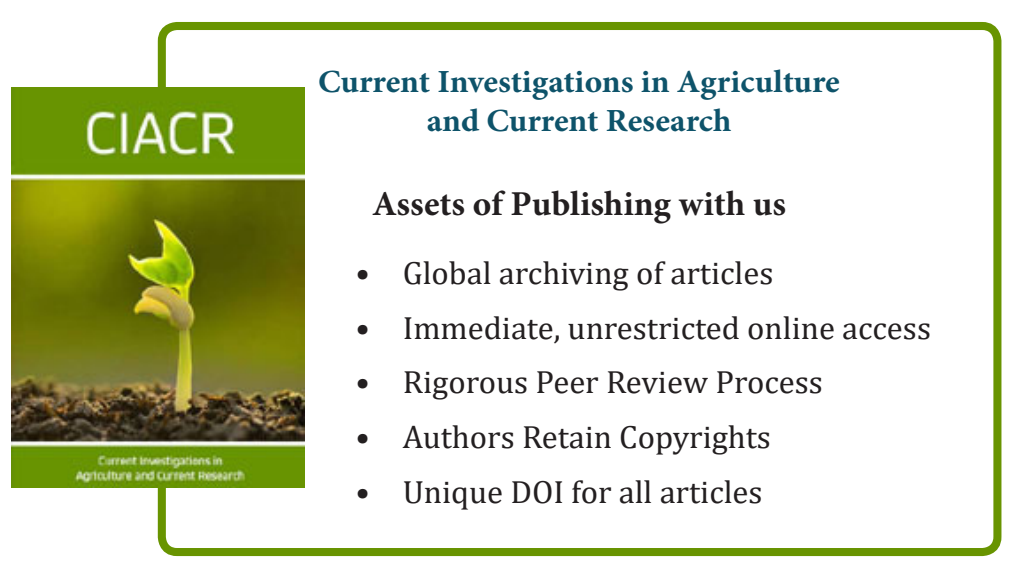

